RAEIC, Revista de la Asociación Española de Investigación de la Comunicación

\title{
Los estudios de audiencia en la formación de los graduados en Comunicación Audiovisual en España
}

\author{
Audiencia studies in the training of graduates in Audiovisual \\ Communication in Spain
}

\author{
González-Neira, Ana \\ Universidade da Coruña (UDC) \\ ana.gneira@udc.es \\ Quintas-Froufe, Natalia \\ Universidade da Coruña (UDC) \\ n.quintas.froufe@udc.es
}

Forma de citar este artículo:

González-Neira, A., y Quintas-Froufe, N. (2020). "Los estudios de audiencia en la formación de los graduados en Comunicación Audiovisual en España", RAEIC, Revista de la Asociación Española de Investigación de la Comunicación, vol. 7, núm. 13, 77-98

https://doi.org/10.24137/raeic.7.13.4

\section{Resumen:}

En un contexto de empoderamiento de las audiencias como el actual, la formación de los futuros profesionales de la comunicación debe tener en cuenta la figura del receptor para abordar los retos que se plantean en el mundo laboral.

RAEIC, Revista de la Asociación Española de Investigación de la Comunicación vol. 7, núm. 13 (2020), 50-98 
El propósito de esta investigación es analizar y contrastar los planes de estudio del grado en Comunicación Audiovisual ofertados por la universidad española para conocer el peso que los estudios de audiencia tienen tienen en dichas enseñanzas. Tras el análisis de las memorias de los títulos y la información facilitada por los responsables de las titulaciones se puede concluir que el número de asignaturas centradas exclusivamente en este campo es minoritario. El estudio de las audiencias aparece visiblemente interrelacionado con la programación televisiva y radiofónica en los diferentes planes de estudio del grado en Comunicación Audiovisual, de ahí que en múltiples ocasiones se incluya como una unidad temática de la asignatura de programación y no tenga entidad propia como asignatura. Otra de las conclusiones que aporta este estudio es que tampoco es habitual que las universidades españolas introduzcan en la denominación de las asignaturas con contenidos vinculados a las audiencias referencias a los estudios de recepción.

Palabras clave: audiencia, universidad comunicación audiovisual recepción.

\section{Abstract:}

In a context of empowerment of audiences such as the current one, the training of future communication professionals must take into account the figure of the recipient to address the challenges that arise in the workplace. The aim of this research is to analyze and contrast the study programmes of the degree in Audiovisual Communication offered by the Spanish university to know the weight that audience studies have in the training of future professionals. After analyzing the report of the degrees and the information provided by those responsible it can be concluded that the number of subjects focused exclusively in this field is a minority. The study of the audiences appears visibly interrelated with the television and radio programming in the different study programmes of the degree in Audiovisual Communication, hence on multiple occasions it is included as a thematic unit of the programming subject and does not have its own entity as a subject. Nor is it common for Spanish universities to introduce references to reception studies in the denomination of subjects with contents linked to the audience. 
Keywords: audience, university, audiovisual communication, reception.

\section{INTRODUCCIÓN}

Los cambios en el ecosistema mediático han favorecido el incremento del protagonismo de las audiencias. Los tradicionales receptores se han convertido en activos usuarios que deciden su dieta mediática y disponen de múltiples posibilidades de interacción a través de las opciones de personalización y participación (Rost, 2004). Los avances tecnológicos y los acuerdos empresariales han propiciado el nacimiento de las audiencias creativas (Deltell, 2014) que participan activamente en el proceso comunicativo. En estos momentos, el usuario ha asumido la interactividad como algo intrínseco de su vida digital, por lo que la participación aparece como elemento esencial de la nueva realidad mediática. El usuario demanda que esa interactividad, con grados de especialización creciente, esté presente en los medios que consume. El espectador de la televisión actual posee un perfil activo, multimedia, multiplataforma, en contacto constante con la red y con las tecnologías de la información y acostumbrado al visionado on line (García García et al. 2012, p.124). Por lo tanto, ante estas prácticas de consumo deben satisfacer una experiencia de usuario cada vez más exigente.

Asimismo, la organización de cualquier proyecto audiovisual exige, de forma previa y también durante el desarrollo del mismo, tener en cuenta a los receptores a los que está destinada. Por lo tanto, los estudiantes del grado en Comunicación Audiovisual deberán poseer conocimientos básicos sobre cómo definir y acotar su público objetivo de sus creaciones. Conceptos como prosumidores, influencers, audiencia social, fandom se han incorporado a la agenda de los estudiosos de la comunicación como elemento clave en el campo que nos ocupa. Esa importancia que han adquirido debe trasladarse a la enseñanza de los futuros profesionales de la comunicación en España para dotarles de conocimientos suficientes en el tratamiento de los receptores de la comunicación. En un proceso de redefinición de los profesionales de la comunicación como el actual es necesario realizar una adaptación de los estudios a las exigencias del mercado laboral. De acuerdo con Tejedor y Cervi (2017) este escenario comunicativo exige "una reflexión 
continuada sobre las habilidades, las competencias y los perfiles que exige la industria" (p. 1627).

Investigaciones previas a esta analizaron el planteamiento global de los estudios universitarios en Comunicación desde diferentes perspectivas (Figueras Maz et al, 2018; Sánchez, Ormaechea y Ramos, 2018; Guarinos, Caro y Durán, 2018; Civil i Serra, 2018; Tejedor y Cervi, 2017; Perlado y Saavedra, 2017; Jurado Martín, 2017; Grandío Pérez, 2016; Lazcano-Peña, 2013; López-Escobar y Martín Algarra, 2016; Gómez Mompart y Herrero Subías, 2015; Pérez Serrano, Cabezuelo y Fanjul, 2010; Sierra Sánchez y Cabezuelo Lorenzo, 2010; Castelló-Martínez, 2012; García Avilés y García-Jiménez, 2009) y más concretamente la docencia universitaria en el ámbito de las audiencias (Piñuel y Mariño, 2016).

\section{CONTEXTUALIZACIÓN}

Los estudios en Comunicación no poseen una larga tradición en Europa, de ahí que su incorporación a la enseñanza superior fuera tardía. El Libro blanco de los Títulos de Grado en Comunicación señala que la antigüedad media de esta disciplina en la universidad hay que situarla entre los 30 y 20 años. Sin embargo, como señaló Rodríguez Merchán (2007), España fue uno de los primeros países de Europa en incorporar los estudios oficiales de cinematografía a la universidad, aunque fue un proceso lento que abarcó desde la década de los sesenta hasta los ochenta.

Las Escuelas Oficiales de Periodismo, Publicidad, Cinematografía y Radiodifusión y Televisión pueden considerarse los antecedentes más inmediatos de la enseñanza reglada de la Comunicación en España ${ }^{1}$ ya que fueron las responsables de formar a los primeros profesionales del sector. Concretamente, la Escuela de Cinematografía creada en 1962 puede calificarse como las precursoras de la titulación de Imagen y Sonido que posteriormente daría lugar a la licenciatura en Comunicación Audiovisual.

\footnotetext{
${ }^{1}$ Con anterioridad existieron otras iniciativas como por ejemplo el curso de periodismo organizado por el catedrático y periodista Fernando Araujo y Gómez en la Universidad de Salamanca en el año 1887, o las Escuelas de Periodismo en las décadas de los años 20 y 30 de El Debate y Mundo Obrero.
} 
Los estudios en Comunicación se fueron consolidando en España de forma progresiva, pero hasta 1971 no adquirieron el rango universitario. El Decreto del Ministerio de Educación y Ciencia 2070/1971, de 13 agosto (RCL 1971/1960), reguló los estudios de periodismo y demás medios de comunicación social. Unos meses después, y al amparo de la Ley General de Educación que reglamentó también el anterior decreto, el mismo Ministerio emitió el Decreto 2478/1971, de 17 septiembre (RCL 1971/1871) que creó las primeras Facultades de Ciencias de la Información en Madrid y Barcelona, en la Universidad Complutense en el primer caso y en la Autónoma en el segundo. Dicho Decreto anunciaba la creación de las Facultades de Ciencias de la Información, estructuradas en departamentos de Periodismo, de Ciencias de la Imagen Visual y Auditiva y de Publicidad, con arreglo a los tres ciclos dispuestos en la Ley. La creación de estas primeras Facultades supuso el cierre definitivo de las Escuelas existentes hasta el momento. Lo que en un principio se agrupó bajo la rama de Ciencias de la Imagen Visual y Auditiva, terminaría dando lugar a la licenciatura en Comunicación Audiovisual y posteriormente al grado en Comunicación Audiovisual.

Como señaló Rodríguez Merchán (2007) la Facultad de Ciencias de la Información de la Universidad Complutense monopolizó la enseñanza de las asignaturas relacionadas con el cine y la televisión. Desde el departamento de Imagen Audiovisual y Auditiva² ${ }^{2}$ dirigido por el primer catedrático del área Antonio Lara García, se trató de paliar la escasa oferta académica pública y privada existente en España en aquel momento (Rodríguez Merchán, 2007).

La creación de estas Facultades constituyó, como indicó Martínez Nicolás (2009), un marco institucional adecuado -o al menos más adecuado- para la emergencia de una comunidad científica. Desde ese momento hasta el actual se produjo una continua creación de nuevas Facultades de Ciencias de la Información y de la Comunicación.

A partir de los años noventa hubo una proliferación de nuevas Facultades de Comunicación que imparten en muchos casos estudios de Comunicación Audiovisual, aprovechando la aprobación de las tres titulaciones de Periodismo, Publicidad y

\footnotetext{
${ }^{2}$ Posteriormente sería el departamento de Comunicación Audiovisual y Publicidad.
} 
Relaciones Públicas y Comunicación Audiovisual (Real Decreto 1427/1991, de 30 de agosto) (RCL 1991/2453), frente a la única que existía anteriormente (Martínez Nicolás, 2009). Paralelo al crecimiento de centros, se produjo una masificación en la demanda de los estudios de Comunicación. Prueba de ello son las altas notas de corte que exigen algunos centros en la actualidad. Por lo tanto, mientras hace unas décadas los estudios en Comunicación Audiovisual eran una especialidad en las titulaciones de Ciencias de la Información, en la actualidad se han convertido en uno de los grados más dinámicos y con mayor demanda de la oferta universitaria española.

\section{LA ADAPTACIÓN DE LOS ESTUDIOS DE COMUNICACIÓN AUDIOVISUAL AL EEES}

En el año 2005 ANECA elaboró un documento denominado Libro blanco de los Títulos de Grado en Comunicación (Periodismo, Comunicación Audiovisual y Publicidad y Relaciones Públicas) con el fin de recopilar todos los aspectos fundamentales que se deben tener en cuenta a la hora de diseñar un título de grado en Comunicación adaptado al Espacio Europeo de Educación Superior. Esta propuesta fue realizada por una red de cuarenta universidades españolas, todas las que impartían alguna de las tres titulaciones, con el fin de encontrar un modelo consensuado por todas.

Una de las primeras recomendaciones indicadas en el libro fue la conveniencia de mantener las tres titulaciones de modo independiente, a pesar de que antes de la reforma de los últimos planes de estudios, el primer ciclo era común en muchos de los centros en los que se impartían las tres carreras. De modo que se propusieron tres titulaciones de grado con una duración de cuatro años (240 ECTS) ${ }^{3}$, "por cuanto estimamos que un período de tiempo menor sería insuficiente para la adquisición de los conocimientos, competencias y destrezas, tanto académicas como profesionales, que los futuros periodistas, comunicadores audiovisuales, publicitarios y relaciones públicas precisan" (ANECA, 2005, p.307).

\footnotetext{
${ }^{3}$ Las Universidades Carlos III, San Pablo CEU, Oberta de Catalunya, Pompeu Fabra (Periodismo) y Valencia se mostraron partidarias de un grado generalista de 180 créditos y un postgrado de perfil profesional de 120 créditos.
} 
Este planteamiento difiere del sostenido en otros países de Europa tales como Francia o el Reino Unido, en los que se ofertan títulos más generalistas, enmarcados bajo el epígrafe de "Estudios de comunicación", "Información y Comunicación" o “Comunicación social” entre otros (López-Berna, Papí-Gálvez y Martín-Llaguno, 2010).

La justificación de la propuesta se articuló sobre la necesidad de impartir una formación especializada a diferentes tipologías profesionales que, aún compartiendo el ámbito común de la Comunicación Social, tienen unos perfiles específicos técnicos y científicos: "todos son comunicadores sociales, pero no todos hacen las mismas cosas ni comparten idénticas funciones comunicativas (que pueden ser parecidas pero no semejantes), y por ello requieren igualmente preparaciones académicas específicas, correctamente diferenciadas" (ANECA, 2005, p.305).

En el documento final se describieron los perfiles profesionales y las competencias de la titulación, las exigencias formativas y los objetivos generales de los estudios en Comunicación Audiovisual, así como la estructura del título y los planes de estudio.

En un principio la titulación se diseñó en función de cuatro perfiles profesionales básicos y cuatro bloques temáticos: 1) Director/a y realizador/a audiovisual, 2) Productor/a y gestor/a audiovisual, 3) Guion audiovisual y 4) Diseño de producción y postproducción visual y sonora.

Sin embargo, finalmente se descartó que el perfil profesional de guion audiovisual fuera independiente (se incorporó al perfil de Director/a y realizador/a audiovisual) y se vio necesario la creación de un perfil propio de investigador, docente y experto en estudios visuales.

El Libro Blanco estableció una propuesta de reparto de contenidos en tres bloques: los contenidos obligatorios comunes (50\%), los contenidos instrumentales obligatorios (10\%) y los contenidos propios de cada Universidad (40\%). 
Los contenidos obligatorios comunes incluían las siguientes materias ${ }^{4}$ : Técnicas y procesos creativos de producción, realización y postproducción, Teoría e historia de los medios audiovisuales y nuevos soportes multimedia, Análisis de los discursos audiovisuales y de sus efectos sociales, Procesos de ideación y narrativa audiovisual, Organización y producción audiovisual y multimedia, Industrias culturales y movimientos estéticos contemporáneos y Estructura y políticas de la comunicación audiovisual. Los contenidos relacionados con el estudio de la audiencia están vinculados a las materias Teoría e historia de los medios audiovisuales y nuevos soportes multimedia, Análisis de los discursos audiovisuales y de sus efectos sociales y Organización y producción audiovisual y multimedia.

Los contenidos vinculados a la audiencia se incorporaron a los planes de estudio de las titulaciones ligadas a la Comunicación, tal como se recomendó en el Libro blanco de los Títulos de Grado en Comunicación. Las titulaciones de Publicidad y Relaciones Públicas fueron las que mayoritariamente ofertaron asignaturas con esta misma denominación, si bien más orientadas a la investigación de la audiencia en el conjunto del proceso publicitario.

\section{METOdOLOGÍA}

En un contexto de audiencias activas (Livingstone, 2004) y conversión del receptor en un prosumidor se hace necesario conocer hasta qué punto los estudios de audiencias se contemplan en una de las titulaciones más dinámicas y demandadas del sistema universitario español. Los retos que plantean los cambios en el mundo laboral y los avances técnicos exigen una adaptación y reconceptualización de los planes de estudios. Por ello, el objetivo de esta investigación es analizar y contrastar qué planes de estudio del grado en Comunicación Audiovisual ofertados en la actualidad por la universidad española incluyen asignaturas con contenidos vinculados al estudio de la audiencia. De esta forma se pretende conseguir una visión global en el ámbito nacional.

\footnotetext{
${ }^{4}$ El orden de las materias responde al número de créditos asignados (de mayor a menor).
} 
Para realizar dicho análisis exploratorio de carácter descriptivo se han estudiado los planes de estudio del grado en Comunicación Audiovisual de todas las universidades españolas (públicas y privadas). Se ha tomado como primera referencia las titulaciones de Comunicación Audiovisual incorporadas al Registro de Universidades, Centros y Títulos (RUCT) del Ministerio de Ciencia, Innovación y Universidades ${ }^{5}$. Posteriormente se procedió a localizar las memorias de los diferentes títulos como documento central que rige los diferentes estudios de grado. La búsqueda de este material marco se realizó tanto a través de las webs de las universidades como mediante el contacto con los diferentes responsables de las titulaciones y/o materias. La memoria del título, el contacto con los responsables del grado y la información disponible en las respectivas páginas web fueron la base de este estudio. De este modo se pudo acceder a todos los contenidos mínimos impartidos en los grados de Comunicación Audiovisual y localizar aquellos relacionados con los Estudios de Audiencia tanto en los descriptores detallados en la memoria como a partir de la información más actualizada en la web.

Se parte de la hipótesis de que la figura del receptor, vinculada a los estudios de audiencia, es transversal a muchas asignaturas en los estudios de Comunicación Audiovisual. En esta investigación se recogieron datos solo de aquellas materias que o bien se centraban íntegramente en los estudios de audiencia y recepción, o bien abordaban parcialmente en algunas de sus unidades didácticas contenidos vinculados a estos estudios. La investigación se realizó siguiendo una metodología mixta en la que se elaboró una ficha de análisis con diversas variables para el registro de datos (centro universitario, nombre de la materia, número de créditos, carácter, curso en la que se imparte, si los contenidos sobre audiencia se tratan de forma exclusivo o si bien solo constituyen alguna unidad didáctica). El trabajo de campo se realizó durante los primeros seis meses de 2019 por lo tanto ofrece resultados para un momento determinado en un contexto de renovación de los planes de estudio de este grado.

\footnotetext{
${ }^{5}$ Agradecemos a la sección de Estudios de Recepción y Audiencias de la Asociación Española de Investigación de la Comunicación su colaboración en esta investigación.
} 


\section{RESULTADOS}

En la actualidad las universidades que ofertan el grado en Comunicación Audiovisual y Comunicación Audiovisual y Multimedia son 40 (24 de ellas de carácter público y 16 privado), según la comprobación realizada tras los datos obtenidos en el Registro de Universidades, Centros y Títulos ${ }^{6}$ del Ministerio de Educación, Cultura y Deporte.

De las 40 universidades que ofertan grados de Comunicación Audiovisual se han localizado 34 que imparten contenidos sobre audiencia (12 de ellas privadas y 22 públicas) según los parámetros explicados previamente en el apartado de metodología?.

Gráfico 1. Universidades que ofrecen materias relacionadas con la audiencia.

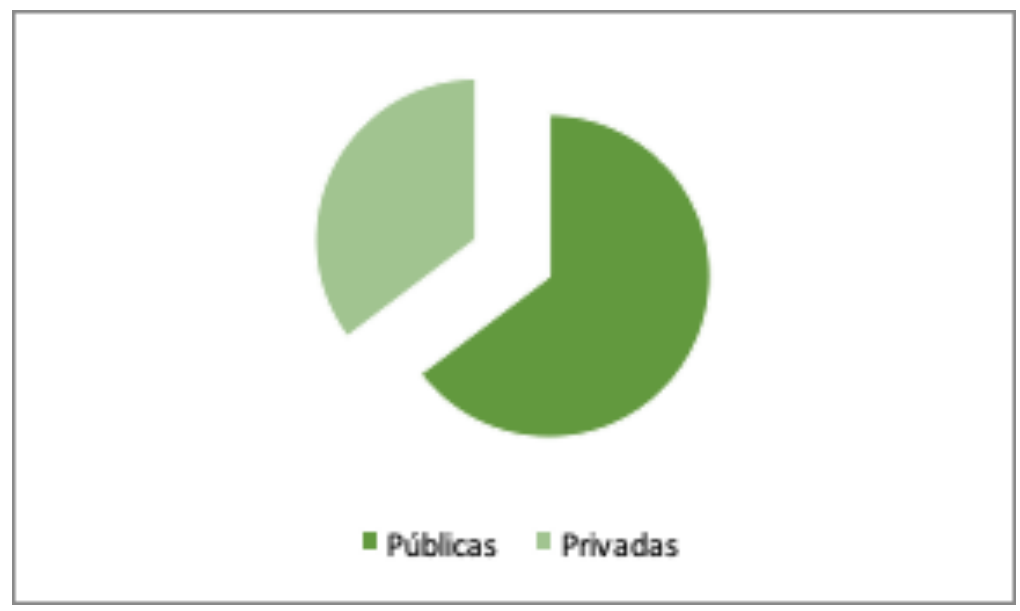

Fuente: Registro de Universidades, Centros y Títulos. Elaboración propia

Este dato supone que el $91 \%$ de las universidades públicas imparten contenidos relacionados, mientras que en el caso de los centros privados, este porcentaje desciende al $75 \%$. No obstante, se concluye que estos contenidos suelen estar presentes en la mayor parte de los grados oficiales de Comunicación Audiovisual impartidos en España.

\footnotetext{
${ }^{6}$ A fecha de 1 de marzo de 2019.

${ }^{7}$ En el momento de recogida de datos algunas universidades como la Autónoma de Barcelona estaba en un proceso de renovación de planes de estudio. El nuevo plan no contempla ninguna asignatura de Audiencias.
}

RAEIC, Revista de la Asociación Española de Investigación de la Comunicación vol. 7, núm. 13 (2020), 77-98 
Gráfico 2. Universidades públicas que ofrecen materias relacionadas con la audiencia.

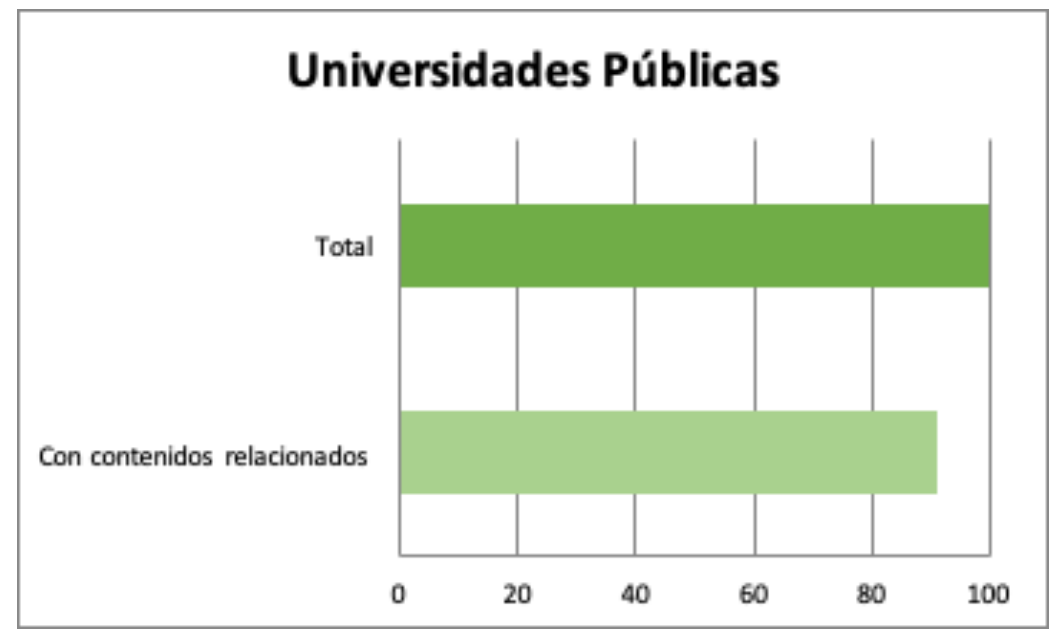

Fuente: Registro de Universidades, Centros y Títulos. Elaboración propia

Gráfico 3. Universidades privadas que ofrecen materias relacionadas con la audiencia.

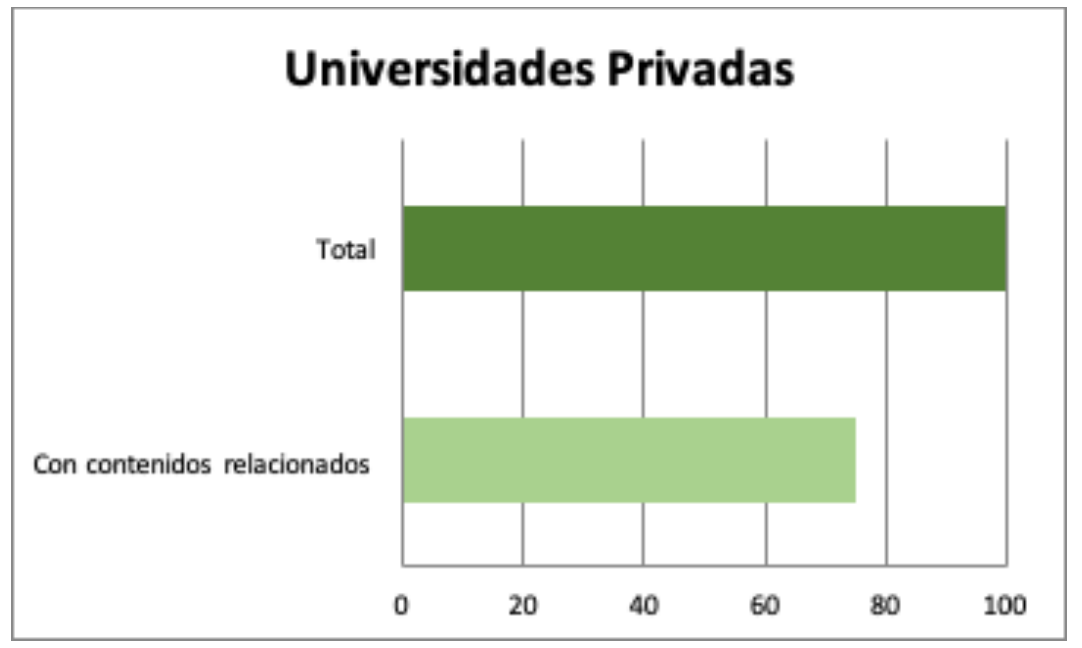

Fuente: Registro de Universidades, Centros y Títulos. Elaboración propia

Tras el análisis realizado se han localizado en los diferentes grados 62 materias que abordan en distinta profundidad contenidos de audiencia. Veinte de ellas son ofertadas por centros privados. Con el fin de conocer el grado de dedicación/profundidad de esas asignaturas, éstas se clasificaron en aquellas que ofrecen íntegramente un temario de audiencias y aquellas en las que este contenido se aborda en alguna unidad didáctica, y por lo tanto no constituye la parte mayoritaria de la asignatura. La investigación desvela que tan solo se ofertan diez materias completamente centradas en los contenidos de audiencia (Universidad Pontificia Comillas-CESAG, Universitat Autónoma de Barcelona, Universidad Carlos III, Universidad Alcalá, Universidad de Salamanca, Universidade de A 
Coruña, Universitat de Vic, Universitat Pompeu Fabra, Universitat Ramón Llull y Universitat Politècnica de València). Es decir, el número de materias íntegramente consagradas a los estudios de audiencia es claramente minoritario dentro de la oferta de asignaturas en los grados de Comunicación Audiovisual y lo ofrecen en su mayoría universidades de titularidad pública.

Gráfico 4. Número de materias en función del grado de profundización en los estudios de audiencia.

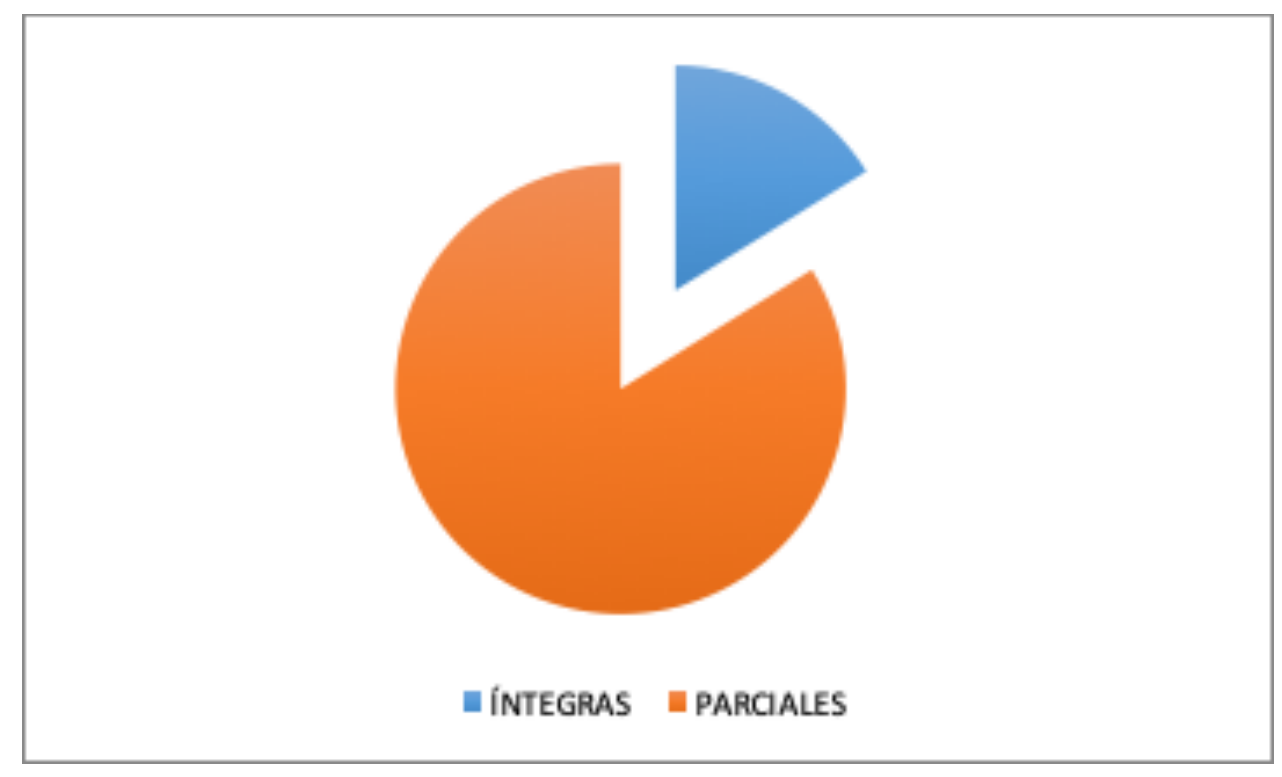

Fuente: Elaboración propia

Las 52 materias restantes ofrecen dichos contenidos incluidos en asignaturas de muy diversa índole. A partir de los datos facilitados y publicados, se han localizado veinte materias sobre Programación audiovisual que dedican al menos una unidad didáctica a cuestiones relacionadas con la audimetría. Asimismo, los contenidos sobre audiencia se incluyen en asignaturas sobre Estructura del sistema audiovisual (Universidad Herrera Oria, Universidad Carlos III, Universitat de Barcelona, Universidad de Murcia, Universidad de Navarra, Universidad de País Vasco, Universitat Rovira i Virgili), Teoría de la Comunicación (Universidad de Málaga, Universitat Jaume I, Universidad Miguel Hernández, Universidad Pontificia de Salamanca), Nuevos formatos o nuevas tendencias de la comunicación (Mondragón Unibertsitatea, Universidad Carlos III y Universitat Ramón Lull), Sociología (Universidad Pontificia Comillas-CESAG, Universidad Católica de Murcia y Universidade de Vigo), Publicidad (Mondragón Unibertsitatea, Universidad 
Carlos III y Universidad de Granada), Estudios televisivos (Universidad Carlos III, Universidad de Burgos, Universidad Europea del Atlántico), Marketing (Universidad Católica de Murcia y Universidad de Granada), así como Historia (Universidad de Nebrija), Cultura digital (Universidad Pontificia Comillas-CESAG) o Ficción (Universidad de Murcia), entre otras.

Por lo que se refiere a la dimensión de la oferta de materias por centros, a partir de los datos analizados se constata que la mayor parte de ellos ofrecen más de una materia vinculada con estos contenidos. Tan solo 15 de estas universidades cuentan con una sola asignatura relacionada con los estudios de audiencias (44\%). Entre los 19 centros restantes, destaca la Universidad Carlos III que cuenta con una oferta mayor al ofrecer siete asignaturas relacionadas con contenidos de audiencia. Tras ella se sitúan la Universidad Pontificia Comillas-CESAG, Mondragón Unibertsitatea, Universidad de Granada y Universidad Miguel Hernández que proponen tres materias.

En cuanto al carácter de las materias, el $61,2 \%$ son obligatorias. Si se desglosa en cuanto al peso del contenido de audiencias se comprueba que el $70 \%$ de aquellas íntegramente dedicadas son obligatorias frente al $59 \%$ de las materias parciales. Ninguna de las asignaturas centradas de forma exclusiva en los estudios de audiencia forma pate de las materias básicas. En cuanto a aquellas parciales el 15\% son de formación básica. Por lo que se refiere a la oferta de optativas, el $25,8 \%$ de las materias objeto de estos pertenecen a esta categoría. Al desglosar los datos comprobamos que el $30 \%$ de las materias centradas en los estudios de audiencia son optativas frente al $25 \%$ de aquellas parciales. 
Gráfico 5. Tipo de materias vinculadas con los contenidos de audiencias.

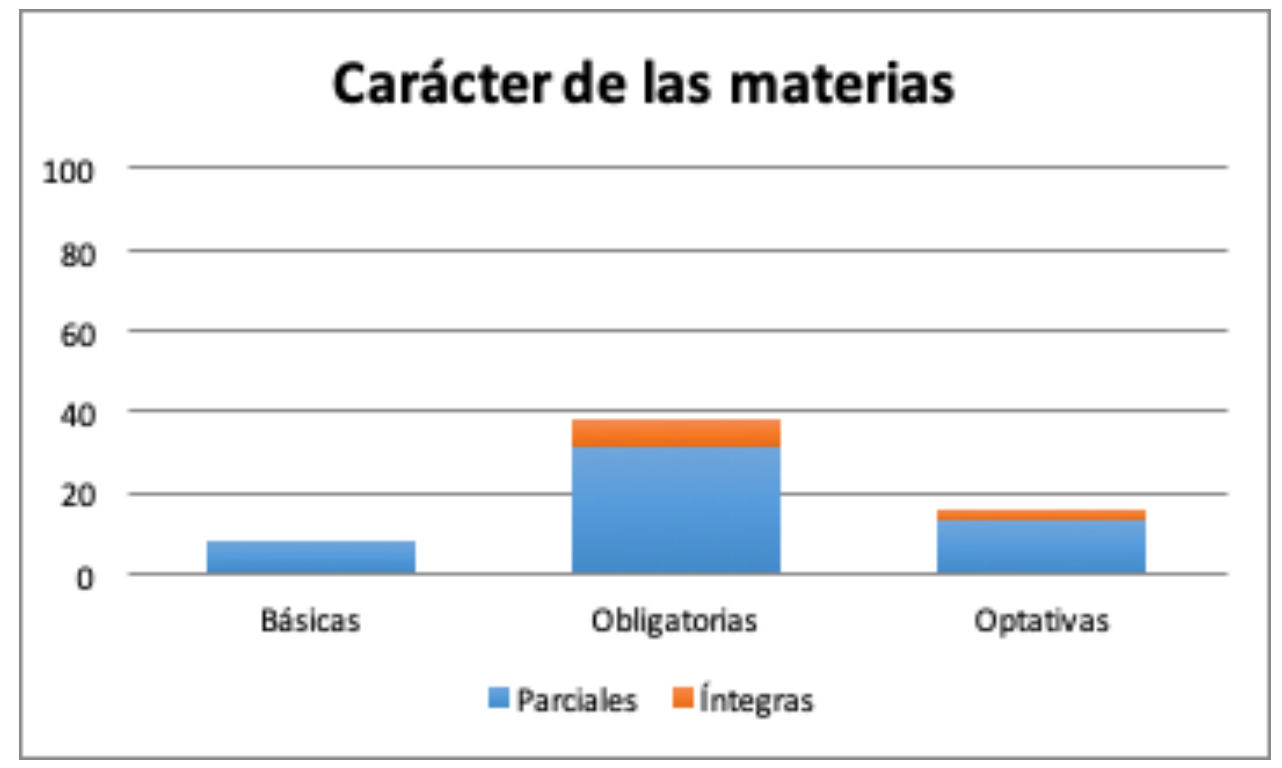

Fuente: Elaboración propia

Este dato demuestra que la mayoría de las universidades consideran que estos contenidos son suficientemente importantes para formar parte de la instrucción obligatoria y del proceso de formación de los universitarios sin dejarla a ser sujeto de la optatividad. Ambas categorías de asignaturas coinciden al situar en su mayoría a estas materias como obligatorias.

Por lo que respecta a su situación en el plan de estudios, las materias relacionadas con las audiencias suelen aparecer en tercer curso principalmente (un $46,7 \%$ ). Una de las razones de este reparto se debe a que, como se indicó anteriormente, algunas de ellas son optativas por lo que se ofertan en los dos cursos finales del grado. En el caso de aquellas materias dedicadas íntegramente a los estudios de audiencia, estas nunca se sitúan en el primer curso de carrera, a diferencia de 8 de aquellas en las que este contenido es parcial.

Se ha estudiado asimismo el número de créditos que tienen las asignaturas relacionadas con el estudio de las audiencias para concluir que el 69,3\% de las materias se ofertan con 6 ECTS, es decir la mayoría de las materias que abordan este tipo de contenidos coinciden en el número de créditos. 
Gráfico 6. Curso en el que se imparten las materias.

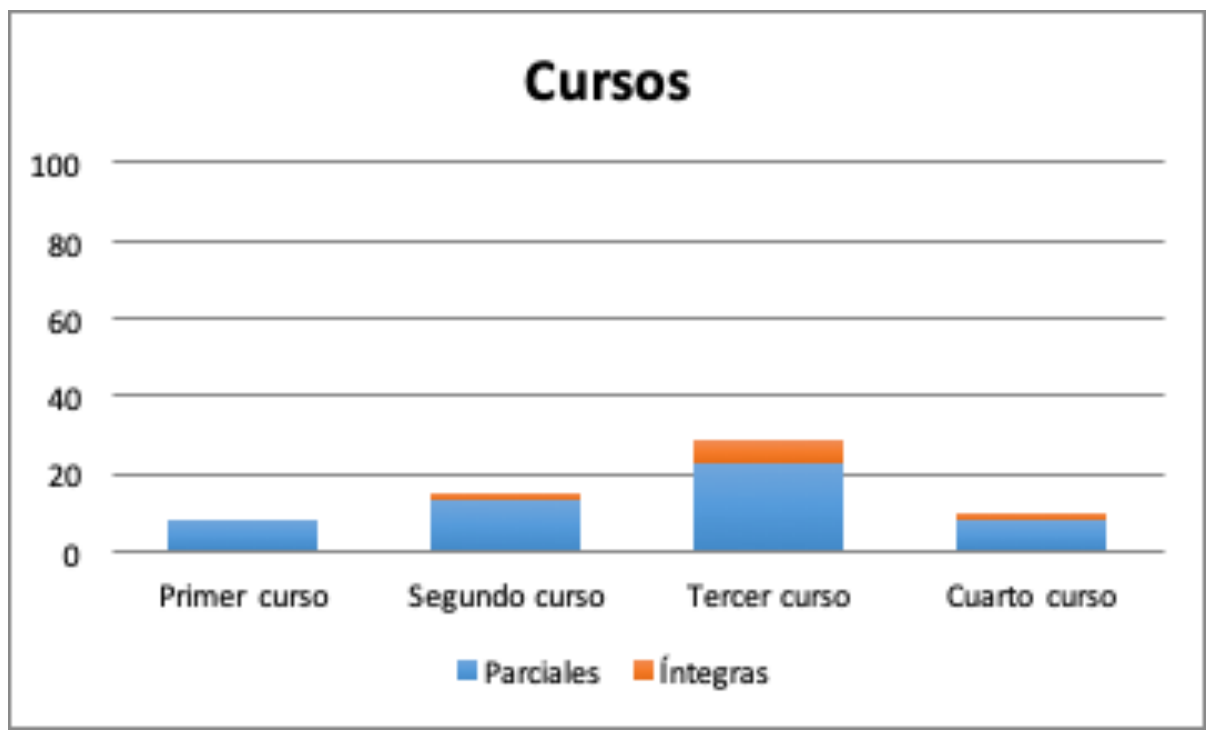

Fuente: Elaboración propia

Gráfico 7. Número de créditos de las materias.

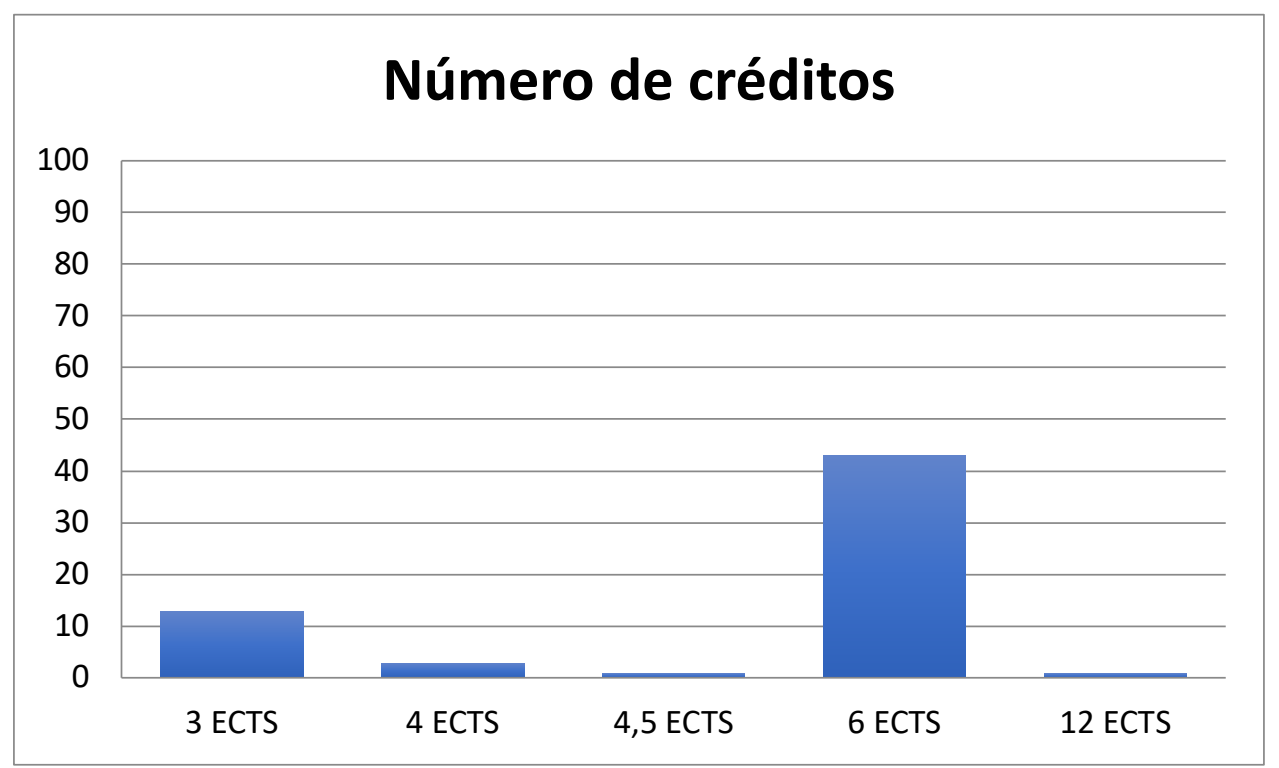

Fuente: Elaboración propia

La mitad de las asignaturas dedicadas íntegramente a los estudios de audiencia tienen este número de créditos, frente al $73 \%$ de aquellas que lo abordan de forma parcial. El 30,9\% tienen 3 ECTS (diferenciados entre 30\% y 19\% en función de los tipos de asignaturas analizados) mientras que tan solo el 4,8 \% tienen 4 ECTS. Cabe destacar que existe solo una sola materia de 4,5 ECTS (Universitat Politècnica de Valncia) y otra de 12 ECTS (Universidad de Murcia). 
Tras la recopilación de todos los datos se procedió a la discusión de los resultados que se abordará en el siguiente epígrafe.

\section{DISCUSIÓN Y CONCLUSIONES}

El análisis realizado permite afirmar que en el $85 \%$ de los grados en Comunicación Audiovisual impartidos en España existe al menos una materia que aborda contenidos de audiencia. Se trata sin duda de un dato elevado. Sin embargo, se ha comprobado que la mayor parte de estas asignaturas tratan este tema de forma parcial al incluirlo como unidad didáctica de una materia y tan solo en diez ocasiones constituyen una asignatura independiente.

No ha sido objeto de esta investigación un estudio pormenorizado del temario y de las competencias de estas asignaturas. No obstante, del análisis realizado se pueden extraer algunas conclusiones generales sobre la perspectiva desde la cual se abordan los contenidos de audiencia en las materias compartidas. En veinte ocasiones algunos de sus contenidos básicos se integran en otras asignaturas relacionadas con la Programación. Por ejemplo, en la Universidad de Extremadura, en la guía docente de la asignatura Programación Audiovisual se incluye una unidad temática sobre la investigación de audiencias en televisión; en la Universidad de Málaga, en el programa de la asignatura Programación Audiovisual se contempla un tema dedicado a las audiencias; en la asignatura de Programación en Radio y Televisión de la Universidad Jaume I se incorpora una unidad temática sobre los públicos y la investigación de audiencias o en la Universidad San Jorge se ofrece la asignatura Programación Televisiva con un tema que versa sobre el cliente-espectador en el que se aborda la cuantificación y medición de la audiencia.

En otras asignaturas centradas en la programación, como por ejemplo Programación y Gestión Televisiva y Radiofónica en la Universidad de Sevilla, presentan también alguna unidad temática sobre la audimetría al igual que, en asignaturas como Programación y Formatos Audiovisuales (Universidad Francisco de Vitoria) donde también se incluye un tema específico sobre la audimetría y la medición de la audiencia en los diferentes medios audiovisuales. 
Cabe destacar, por ser casos claramente excepcionales, la Universidad Pompeu Fabra, la Universidad de Alcalá y la Universidad de Salamanca que ofrecen en sus planes de estudio dos asignaturas independientes para abordar la audiencia y la programación en radio y televisión:

- En la Universidad Pompeu Fabra: Investigación en Audiencias y Políticas de Programación.

- En la Universidad de Alcalá: Gestión de contenidos y programación audiovisual e Investigación de audiencias.

- En la Universidad de Salamanca: Estrategias de Programación en Radio y Televisión e Investigación de Audiencias.

Sin embargo, el peso de las asignaturas en el conjunto del plan de estudios no es igual ya que en la Universidad de Salamanca y la Universidad de Alcalá las dos asignaturas son obligatorias mientras que en la Pompeu Fabra ambas son optativas.

Tampoco es habitual que las universidades españolas incluyan en la denominación de las asignaturas con contenidos vinculados a las audiencias referencias a los estudios de recepción, algo que únicamente sucede en la Universidad de Santiago de Compostela con la asignatura Programación y Análisis de la Recepción y en la Universidad Autónoma de Barcelona con Estudios de Audiencia y Recepción.

Por último, cabe destacar que el concepto de público aparece ligado, de forma explícita o implícita, al de audiencias en la denominación de las asignaturas Públicos y Audiencias de la Universidad Pontificia de Comillas y El Público Audiovisual, impartida en el grado bilingüe en Comunicación Audiovisual de la Universidad Carlos III.

Si bien no fue objeto de análisis exhaustivo el estudio de los contenidos de cada materia, sí se puede avanzar que la mayor parte están centradas en el medio televisivo, con escasa atención a la radio o al entorno digital. Estas asignaturas se suelen centrar en técnicas de audimetría dejando al margen aspectos relacionados con los estudios de recepción o teoría de la audiencia. 
En un contexto de protagonismo o de las audiencias motivado por las consecuencias de la digitalización en el actual ecosistema mediático tras la digitalización llama la atención que el estudio de las audiencias en los grados de Comunicación Audiovisual no se contemple como un área funcional independiente de forma mayoritaria ya que solo el $16 \%$ de las materias que lo abordan lo hacen íntegramente sin compartir temario con otras disciplinas. A la vista de las aportaciones de este artículo se comprueba que los contenidos impartidos están visiblemente interrelacionados con la programación televisiva y radiofónica en los diferentes planes de estudio del grado en Comunicación Audiovisual en España, de ahí que en múltiples ocasiones se incluya como una unidad temática de la asignatura de programación y no tenga entidad propia como materia.

Los cambios en el ecosistema mediático obligarán a los responsables de las titulaciones a actualizar constantemente estas materias para contemplar el progresivo empoderamiento de las audiencias en el proceso comunicativo. De hecho, en algunos grados existen ya asignaturas dedicadas a nuevas tendencias de la comunicación en las que se abordan cuestiones como el protagonismo de las audiencias en el panorama mediático actual. Este sería el caso de Generación de nuevos formatos y contenidos en Mondragon Unibertsitatea, Nuevas formas publicitarias y Nuevas tendencias de la comunicación audiovisual en la Universidad Carlos III, Nuevos Medios en la Universitat Ramón Llull, entre otras. En una cultura de la participación como la actual (Jenkins, Ito, Boyd, 2015) los estudios que abordan al receptor como agente activo del proceso de la comunicación son imprescindibles en la formación de los futuros protagonistas de la comunicación audiovisual en España. Estos planes de estudio deben adaptarse a las necesidades del mercado sin perder de vista las bases teórica que sustentan cualquier conocimiento académico. De este modo se irán adaptando los contenidos ofertados con las necesidades reales del mundo profesional para facilitar su inserción laboral.

Los resultados obtenidos en este estudio se podrían completar en un futuro con una investigación sobre las competencias, sistemas de evaluación y/o ejercicios prácticos de estas asignaturas, así como ampliarlo al resto de los grados de comunicación o incluso abordar una perspectiva internacional. De esta forma, se obtendría un panorama 
general sobre la presencia de los contenidos de audiencia en la enseñanza universitaria de primer ciclo en Comunicación mucho más amplia.

\section{REFERENCIAS BIBLIOGRÁFICAS}

Agencia Nacional de Evaluación de la Calidad y Acreditación (ANECA) (2005). Libro blanco de los Títulos de Grado en Comunicación. Disponible en http://www.aneca.es/var/media/150336/libroblanco comunicacion def.pdf

Castelló-Martínez, A. (2012). El estudio de la comunicación publicitaria online en el Grado en Publicidad y RR. PP. Questiones publicitarias, 17, 1-23. Disponible en https://rua.ua.es/dspace/bitstream/10045/34463/1/2012_Castello_QuestionesPublici tarias.pdf

Civil i Serra, M. (2018). Los estudios universitarios de comunicación en España (2017). Análisis de la situación actual para un debate abierto sobre su futuro estratégico. En I. Postigo y M. J. Recorder, (coords). Los y las tics en los estudios de comunicación (pp.1583). Asociación Española de Universidades con Titulaciones de Información y Comunicación, ATIC.

Deltell Escolar, L. (2014). Audiencia social versus audiencia creativa: caso de estudio Twitter. Estudios sobre el mensaje periodístico, 20(1), 33-47.

http://dx.doi.org/10.5209/rev ESMP.2014.v20.n1.45217

Figueras Maz, M., Hernández Escolano, C., Jiménez-Morales, M., Medina Bravo, P., García Cortés, B., Pedraza, R., y Palau i Riberaygua, J. M. (2018). Estudios de Comunicación en la UPF en clave de innovación social: iniciativas docentes con metodología Aprendizaje-Servicio en grado y máster. En V. Martínez Lozano, N. Melero Aguilar, E. Ibañez Ruiz del Portal, M.C. Sánchez Sánchez (eds.). El Aprendizaje-Servicio en la Universidad: una metodología docente y de investigación al servicio de la justicia social y el desarrollo sostenible (pp.76-79). Salamanca: Comunicación Social. 
García García, A. et al. (2012). Nuevas fórmulas de producción audiovisual atendiendo a criterios de interactividad. En L. Bernardino (ed.) La televisión ante el desafío de internet (pp. 122-129). Salamanca: Comunicación Social.

García-Avilés, J. A., y García-Jiménez, L. (2009). La enseñanza de Teorías de la Comunicación en España: análisis y reflexión ante la Convergencia de Bolonia. ZER: Revista de Estudios de Comunicación, 14(27), 271-293

Gómez Mompart, J. L., y Herrero Subías, M. (coord.) (2006). Jornadas de Formación y Empleo en Comunicación. Madrid: Asociación Española de Universidades con Titulaciones de Información y Comunicación.

Grandío-Pérez, M. (2016). El transmedia en la enseñanza universitaria. Análisis de las asignaturas de educación mediática en España (2012-2013). Palabra clave, 19(1), 85104.

Guarinos, V., Caro, F. J., y Durán, S. C. (2018). La igualdad de género en los estudios de grado en Comunicación: la transversalidad imaginaria. Revista Prisma Social, 22, 296325.

Jenkins, H, Ito, M., y Boyd, D. (2015). Participatory culture in a networked era: a conversation on youth, learning, commerce, and politics, Cambridge: Polity Press, Jurado Martín, M. (2017). Innovación en proyectos transversales: optimización de competencias y resultados de aprendizaje en el grado en comunicación audiovisual. En Padilla Castillo (coord.) Aulas virtuales, fórmulas y prácticas (pp. 285-299). McGraw Hill Education.

Lazcano-Peña, D. (2013). Metodologías de investigación: aproximación a su enseñanza en el grado de periodismo en España. Periodística: revista acadèmica, 15, 11-28.

Livingstone, S. (2004). The challenge of changing audiences: Or, what is the audience researcher to do in the age of the Internet? European Journal of Communication, 19(1), 75-86. https://doi.org/10.1177\%2F0267323104040695 
López-Berna, S., Papí-Gálvez, N., Martín-Llaguno, M. (2010). Los estudios de comunicación y el ejercicio profesional en España ante la convergencia europea. En J. Sierra Sánchez (coord.): Los estudios de Ciencias de la Comunicación en el EEES (pp. 133-139). Madrid: Fragua.

López-Escobar, E., y Martín Algarra, M. (2017). Communication teaching and research in Spain. Publizistik, 62(1), 83-105. Disponible en

https://www.mapcom.es/media/files/Communication Teaching and Research in Sp ain.pdf

Martínez Nicolás, M. (2009). La investigación sobre comunicación en España. Evolución histórica y retos actuales. Revista latina de comunicación social, 12(64), 1-14.

Disponible en

http://www.revistalatinacs.org/09/art/01 80001 investigacion/latina art800.pdf

Perlado, M., y Saavedra, M. (eds.) (2017). Los estudios universitarios especializados en Comunicación en España. Un análisis desde la perspectiva profesional de la Dirección de Comunicación (2014-2015). Barcelona: UOC.

Pérez Serrano, M. J., Cabezuelo Lorenzo, F., y Fanjul Peyró, C. (2010). El sistema 'ECTS' y el mapa de competencias como elementos vertebradores de los nuevos estudios de grado en comunicación. Quaderns digitals: Revista de Nuevas Tecnologías y Sociedad, (61), 15.

Piñuel, J. L., y Mariño, V. (2016). Studying audiences in Spain: theoretical and methodological considerations in scholarly current research practices. Presentado en el congreso IAMCR Conference. 29 July 2016 Leicester (UK).

Sánchez, J. S., Ormaechea, S. L., y Ramos, B. L. (2018). Análisis de la materia Trabajos de Fin de Grado (TFG) en los grados de Ciencias de la Comunicación en España. Revista española de Documentación Científica, 41(4), e220.

https://doi.org/10.3989/redc.2018.4.1561 
Rodríguez Merchán, E. (2007). La enseñanza del cine en España: perspectiva histórica y panorama actual. Comunicar, 29, 13-20. https://doi.org/10.3916/26003

Rost, A. (2004) Pero, ¿'De qué hablamos cuando hablamos de Interactividad? Center for Civic journalism, 2, 1-16.

Sierra Sánchez, J., y Cabezuelo, F. (2010). Recursos de las facultades de comunicación para la formación en competencias en el marco del Espacio Europeo de Educación Superior. Doxa Comunicación, 11, 31-54.

Tejedor Calvo, S., y Cervi, L. (2017). Análisis de los estudios de Periodismo y Comunicación en las principales universidades del mundo. Competencias, objetivos y asignaturas. Revista Latina de Comunicación Social, 72, 1626-1647. http://www.revistalatinacs.org/072paper/1238/88es.html 